\title{
The Scandinavian multicenter hemodynamic evaluation of the SJM Regent aortic valve
}

\author{
Jon Offstad ${ }^{1}$, Kai Andersen ${ }^{1}$, Per Paulsson², Jesper Andreasson³ , Ulf Kjellman ${ }^{4}$ Oluf Lundblad ${ }^{5}$, \\ Karl Gunnar Engstrøm ${ }^{6}$, Rune Haaverstad ${ }^{7}$ and Jan L Svennevig ${ }^{1 *}$
}

\begin{abstract}
Background: 112 patients who received small and medium sized St.Jude Regent heart valves $(19-25 \mathrm{~mm})$ at 7 Scandinavian centers were studied between January 2003 and February 2005 to obtain non-invasive data regarding the hemodynamic performance at rest and during Dobutamine stress echocardiography (DSE) testing one year after surgery.
\end{abstract}

Material and methods: 46 woman and 66 men, aged $61.8 \pm 9.7$ (18-75) years, were operated on for aortic regurgitation (17), stenosis (65), or mixed dysfunction (30). Valve sizes were $19 \mathrm{~mm}$ (6), $21 \mathrm{~mm}$ (33), $23 \mathrm{~mm}(41), 25$ $\mathrm{mm}$ (30). Two patients receiving size 27 valves were excluded from the hemodynamic evaluation. Pledgets were used in 100 patients, everted mattress in 66 and simple interrupted sutures in 21. Valve orientation varied and was dependent on the surgeons' choice. 34 patients (30.4\%) underwent concomitant coronary artery surgery.

Results: There were two early deaths (1.8\%) and three late deaths, one because of pancreatic cancer. Late events during follow-up were: non structural dysfunction (1), bleeding (2), thromboembolism (2). At one year follow up 93\% of the patients were in NYHA classes $1-2$ versus $47.8 \%$ preoperatively.

Dobutamine stress echocardiography (DSE) was performed in a total of 66 and maximal peak stress was reached in 61 patients.

During DSE testing, the following statistically significant changes took place: Heart rate increased by $73.0 \%$, cardiac output by $85.5 \%$, left ventriclular ejection fraction by $19.6 \%$, and maximal mean prosthetic transvalvular gradient by $133.8 \%$, whereas the effective orifice area index did not change.

Left ventricular mass fell during one year from $215 \pm 63$ to $197 \pm 62 \mathrm{~g}(\mathrm{p}<0.05)$.

Conclusion: The Dobutamine test induces a substantial stress, well suitable for echocardiographic assessment of prosthesis valve function and can be performed in the majority of the patients. The changes in pressure gradients add to the hemodynamic characteristics of the various valve sizes.

In our patients the St. Jude Regent valve performed satisfactory at rest and under pharmacological stress situation.

Keywords: Aortic valve replacement, Mechanical heart valve, Cardiac function

\section{Introduction}

The current increase in life expectancy implies increasing need for small and medium size valve replacement since the augmented number of aortic stenosis in the elderly is frequently accompanied by a narrow aortic root. Optimal hemodynamic performance of the valve prosthesis is particularly important in this setting [1,2].

\footnotetext{
* Correspondence: j.l.svennevig@medisin.uio.no

'Oslo University Hospital, Rikshospitalet, PO box 4950, Nydalen, NO 0424 Oslo, Norway

Full list of author information is available at the end of the article
}

Bileaflet mechanical valves are considered good longterm substitutes, but are still undergoing improvements. As for their assessment, dobutamine stress echocardiography (DSE) has emerged as a measure of the valve hemodynamics adding to the information obtained at rest [3-12]. However, its diagnostic role in this context needs to be further defined.

The purpose of the present multicenter study was to investigate the performance of small and intermediate sizes of the St. Jude Medical Regent aortic valve, with respect to EOA at various stress levels. This prosthesis

\section{() Biomed Central}


represents the recent generation of a bileaflet mechanical valve. This would, along with previous results [13], contribute to a large scale assessment. Furthermore, the study investigated the hemodynamic response to dobutamine stress related to the size of normally functioning valves. The responses might supplement their hemodynamic characteristics which need to be assessed before an abnormal response to stress can be defined.

\section{Material and methods Patient Population}

A total of 112 patients (46 women and 66 male) undergoing surgery for aortic stenosis $(n=65)$, aortic regurgitation $(\mathrm{n}=17)$ or both $(\mathrm{n}=30)$ between January 2003 and February 2005 at seven Scandinavian centers were included in the study. Valve sizes were $19 \mathrm{~mm}$ (6), 21 $\mathrm{mm}$ (33), $23 \mathrm{~mm}$ (41), $25 \mathrm{~mm}$ (30). Two patients receiving size 27 valves were excluded from the hemodynamic evaluation. Pledgets were used in 100 patients, everted mattress in 66 and simple interrupted sutures in 21. Valve orientation varied and was dependent on the surgeons' choice. 34 patients $(30.4 \%)$ underwent concomitant coronary artery surgery. Concomitant coronary artery bypass surgery was performed in 34 patients. The age was $61.8 \pm 9.7$ years (mean-SD), range 18-75 years. Clinically, 99 patients could be evaluated at follow-up one year following surgery: two patients were excluded due to valve size $27 \mathrm{~mm}$, there were two early and three late deaths, two patients developed Hodgkin's disease and there were missing data in four patients.

\section{Study design}

Standard echocardiographic and Doppler examinations were performed preoperatively, at discharge and one year after the operation at each of the seven centers. In addition, DSE was performed at five centers one year after the operation.

\section{Echocardiographic recordings}

Recordings in the parasternal view were obtained of left ventricular size with measurements of intraventricular septum and posterior wall thickness at end-diastole. Left ventricular outflow tract diameter was reported as the dimension just below the prosthetic valve during early systole. Peak and mean velocities in the left ventricular outflow tract was measured from the apical position. Cardiac output was calculated as: $\mathrm{CO}(\mathrm{l} / \mathrm{min})=\mathrm{VTI}$

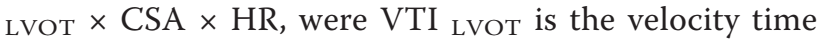
integral in the left ventricular outflow tract, CSA (ot) is the left ventricular outflow tract cross section area, and $\mathrm{HR}$ is heart rate.

Additionally flow velocities across the valve were measured. The prosthetic effective orifice area was calculated as: EOA $\left(\mathrm{cm}^{2}\right)=\mathrm{CSA}_{(0 \mathrm{t})} \times \mathrm{VTI}_{(\mathrm{ot})} / \mathrm{VTI}_{\text {ao }}$.
EOA index $:$ EOA/BSA $\left(\mathrm{cm}^{2} / \mathrm{m}^{2}\right)$. Performance index was calculated as: $\mathrm{PI}=\mathrm{EOA} / \mathrm{MOA}$. $\mathrm{MOA}$ is manufacture orifice area.

\section{Dobutamine stress protocol}

A standard Dobutamine stress echocardiography (DSE) protocol was performed according to the Mayo clinic protocol (14), starting at a Dobutamine infusion rate of $5 \mu \mathrm{g} / \mathrm{kg} / \mathrm{min}$ body weight. The dosage was increased every 5 minutes to $10,15,20,25$ and $30 \mu \mathrm{g} / \mathrm{kg} / \mathrm{min}$. Dobutamine stress was discontinued according to established criteria [14]. Blood pressure and 12- lead electrocardiograms were recorded at baseline and at the end of each stage. Images were obtained in the standard parasternal (long and short axis) and apical (2-4-5 chamber and long axis) views. Standard 2 dimension, M mode and Doppler views were recorded at baseline and at the end of the stress protocol.

The Dobutamine stress test was performed whenever available.

\section{Statistical analysis}

Data are presented as mean \pm SD. Analysis of variance for repeated measurements were performed with valve size as group factors and time (i.e. before and after the operation or before and during stress). Student $t$ tests for comparison of groups and for comparison of paired data were used whenever appropriate. $\mathrm{P}<0.05$ was considered significant.

\section{Consent}

Written informed consent to participate in this study was obtained from each patient. A written consent is available for review by the Editot-in-Chif of this journal. The study was approved by the Regional ethical committees.

\section{Results}

There were two early deaths (1.8\%). One patient was reoperated for bleeding. The mean follow-up time was $12.3 \pm 6.0$ months. During follow- up three patients died, one due to a pancreatic carcinoma. Late events during follow-up were: non structural dysfunction (1), bleeding (2), thromboembolism (2).

At follow up, 93 out of 99 patients (94\%) were in NYHA classes' 1 -2, versus $47.8 \%$ preoperatively. Echocardiographic data before surgery, at discharge and one year after operation is presented in Tables 1 and 2. Left ventricular mass was significantly reduced $(\mathrm{p}<0.05)$ after valve replacement, while no significant changes were observed in left ventricular dimensions or ejection fraction $(\mathrm{EF})$. The transvalvular mean pressure gradient was markedly reduced. The only mild residual mean gradients demonstrated a decrease with increasing valve 
Table 1 Left ventricle dimensions, mass and ejection fraction preoperatively, at discharge and after one year

\begin{tabular}{lllll}
\hline & Preoperatively & Discharge & One year & $\mathbf{p}$ \\
\hline LVDD $(\mathrm{mm}):$ & $53 \pm 9$ & $51 \pm 10$ & $50 \pm 7$ & $\mathrm{~ns}$ \\
IVD $(\mathrm{mm}):$ & $13 \pm 2$ & $8 \pm 1$ & $9 \pm 1$ & $\mathrm{~ns}$ \\
LVPW (mm): & $11 \pm 2$ & $12 \pm 2$ & $11 \pm 2$ & $\mathrm{~ns}$ \\
Mass (g): & $265 \pm 85$ & $260 \pm 95$ & $214 \pm 63$ & $<0.05$ \\
Mass/BSA $\left(\mathrm{g} / \mathrm{m}^{2}\right):$ & $139 \pm 42$ & $137 \pm 43$ & $112 \pm 30$ & $<0.05$ \\
EF (\%): & $55.5 \pm 10.8$ & $54.3 \pm 12.0$ & $58.4 \pm 9.5$ & $\mathrm{~ns}$
\end{tabular}

Mean \pm SD, LVDD; left ventricle diastolic dimension, IVD; intraventricular septum diastolic dimension, LVPW; left ventricle posterior wall dimension, mass; left ventricle mass, Mass/BSA; left ventricle mass index, EF; left ventricle ejection fraction.

size; $11.4 \pm 6.2 \mathrm{mmHg}, 9.2 \pm 6.6 \mathrm{mmHg}, 7.7 \pm 3.3$ $\mathrm{mmHg}$ and $6.0 \pm 2.8 \mathrm{mmHg}$ in the sizes of 19, 21, 23 and $25 \mathrm{~mm}$, respectively. Their corresponding EOA indices were $1.0 \pm 0.4,1.0 \pm 0.2,1.0 \pm 0.3$ and $1.4 \pm$ $0.6 \mathrm{~cm}^{2} / \mathrm{m}^{2}$.

DSE was performed in 66 patients. There were no serious complications. Maximal peak stress was reached in 61 patients $(93 \%)$. The test had to be stopped before peak stress was reached in five patients, due to arrhythmia, chest pain or decrease in blood pressure.

The data obtained during DSE are presented in tables 3 and 4. During DSE heart rate increased on average by $73 \%, \mathrm{CO}$ by $85.5 \%$ and the mean transvalvular gradient by $134 \%$, whereas the EOA did not change significantly.

The gradient at peak stress varied according to valve size whereas EOA index did not.

\section{Discussion}

Hemodynamic properties of small sized aortic prostheses have been questioned, due to the possibility of various degrees of hemodynamic functional obstruction $[1,2]$. Valve prostheses may demonstrate acceptable hemodynamic performance at rest. Additionally, a rise in stroke volume during exercise may increase the pressure drop across the valve and unmask suboptimal valvular function. The hemodynamic performance of aortic valve replacement should therefore be evaluated under various cardiac flow conditions. In this multicenter
Table 3 Hemodynamic variables at rest and during maximal stress $(n=61)$

\begin{tabular}{llll}
\hline & Rest & Peak stress & $\mathbf{p}$ \\
\hline Heart rate $($ beat $/ \mathrm{min}):$ & $63 \pm 11$ & $109 \pm 21$ & $<0.001$ \\
CO $(\mathrm{I} / \mathrm{min}):$ & $5.5 \pm 1.4$ & $10.2 \pm 3.2$ & $<0.001$ \\
Peak gradient $(\mathrm{mmHg}):$ & $15.9 \pm 9.2$ & $38.3 \pm 19.8$ & $<0.001$ \\
Mean gradient $\mathrm{mmHg}):$ & $8.0 \pm 4.8$ & $18.7 \pm 10.0$ & $<0.001$ \\
EOA $\left(\mathrm{cm}^{2}\right):$ & $2.0 \pm 0.7$ & $2.2 \pm 0.8$ & $\mathrm{~ns}$ \\
EOA index $\left(\mathrm{cm}^{2} / \mathrm{m}^{2}\right):$ & $1.1 \pm 0.4$ & $1.2 \pm 0.4$ & $\mathrm{~ns}$ \\
Performance index: & $0.55 \pm 0.16$ & $0.59 \pm 0.20$ & $\mathrm{~ns}$ \\
Ejection fraction $(\%):$ & $57.8 \pm 10.5$ & $69.1 \pm 8.6$ & $<0.001$ \\
\hline
\end{tabular}

study hemodynamic evaluation of small mechanical prostheses in the aortic position was performed by transthoracic echocardiography at rest and during DSE.

Stress echocardiography is the combination of 2D echocardiography with a physical, pharmacological or electrical stress $[15,16]$. Stress echocardiography has been regarded as an important tool in the field of noninvasive diagnosis of coronary artery disease $[17,18]$ and in patients with suspected severe aortic stenosis with low aortic gradients secondary to low cardiac output $[19,20]$.

DSE has additionally been used to examine patients with aortic valve replacement [3-12], mitral valve replacement [20] and double valve replacement. In patients undergoing aortic valve replacement, DSE is an accurate, safe and readily available method to evaluate prostheses hemodynamics [3-12] and to monitor the expected left ventricular hypertrophy regression [12].

The present study demonstrates that DSE can be safely performed in patients one year following heart valve replacement. We used a standard Mayo protocol [14], starting Dobutamine infusion at $5 \mu \mathrm{g} / \mathrm{kg} / \mathrm{min}$. In some previous studies, Dobutamine has been administered to a maximum dose of $20 \mu \mathrm{g} / \mathrm{kg} / \mathrm{min}[3,4,6,7]$. In most of the recent studies a maximum of $40 \mu \mathrm{g} / \mathrm{kg} / \mathrm{min}$ was most often used. Co-administration with atropine [15] has not been used in the studies evaluating prosthesis hemodynamics.

Table 2 Hemodynamic variables preoperatively, at discharge and after one year

\begin{tabular}{|c|c|c|c|c|}
\hline & Preoperatively & Discharge & One year & $\mathrm{p}$ \\
\hline Peak gradient $(\mathrm{mmHg})$ : & $71.7 \pm 32.6$ & $16.8 \pm 9.1$ & $5.9 \pm 9.0$ & $<0.01$ \\
\hline Mean gradient $\mathrm{mmHg}$ ): & $47.9 \pm 22.8$ & $8.7 \pm 5.0$ & $8.0 \pm 4.8$ & $<0.01$ \\
\hline $\mathrm{EOA}\left(\mathrm{cm}^{2}\right)$ & $1.6 \pm 1.8$ & $2.0 \pm 0.7$ & $2.0 \pm 0.7$ & ns \\
\hline EOA index $\left(\mathrm{cm}^{2} / \mathrm{m}^{2}\right)$ : & $0.8 \pm 1.0$ & $1.1 \pm 0.4$ & $1.1 \pm 0.3$ & ns \\
\hline Performance index: & $0.3 \pm 0.2$ & $0.6 \pm 0.2$ & $0.6 \pm 0.2$ & $<0.01$ \\
\hline $\mathrm{CO}(\mathrm{l} / \mathrm{min})$ & $5.9 \pm 1.6$ & $5.8 \pm 2.0$ & $5.5 \pm 1.4$ & $<001$ \\
\hline $\mathrm{Cl}\left(\mathrm{I} / \mathrm{min} / \mathrm{m}^{2}\right):$ & $3.1 \pm 0.8$ & $3.1 \pm 1.0$ & $2.9 \pm 0.6$ & 0.01 \\
\hline HR (beat/min): & $70 \pm 11$ & $81 \pm 13$ & $65 \pm 13$ & $<0.01$ \\
\hline
\end{tabular}

EOA: effective orifice area, EOA index; effective orifice area index, $\mathrm{CO}$; cardiac output, $\mathrm{Cl}$; cardiac index, HR; heart rate. 
Table 4 Mean pressure gradient, effective orifice area (EOA) and effective orifice area index (EOA index) at rest and during peak Dobutamine stress related to valve area

\begin{tabular}{|c|c|c|c|c|c|}
\hline Valve size: & 19 & 21 & 23 & 25 & \\
\hline$(n):$ & (4) & (18) & (23) & (16) & \\
\hline Mean pressure $(\mathrm{mmHg})$ : & & & & & p: \\
\hline At rest: & $11.4 \pm 6.2$ & $9.2 \pm 6.6$ & $7.7 \pm 3.3$ & $6.0 \pm 2.8$ & n.s \\
\hline Peak stress: & $27.3 \pm 22.0$ & $21.1 \pm 10.0$ & $16.9 \pm 8.5$ & $16.7 \pm 5.8$ & n.s \\
\hline \multicolumn{6}{|l|}{ EOA $\left(\mathrm{cm}^{2}\right)$ : } \\
\hline At rest: & $1.6 \pm 0.6$ & $1.8 \pm 0.4$ & $1.8 \pm 0.5$ & $2.8 \pm 1.0$ & $<0.001$ \\
\hline Peak stress: & $2.2 \pm 1.5$ & $1.9 \pm 0.4$ & $1.8 \pm 0.5$ & $2.7 \pm 0.7$ & $<0.02$ \\
\hline \multicolumn{6}{|l|}{ EOA index $\left(\mathrm{cm}^{2} / \mathrm{m}^{2}\right)$ : } \\
\hline At rest: & $1.0 \pm 0.4$ & $1.0 \pm 0.2$ & $1.0 \pm 0.3$ & $1.4 \pm 0.6$ & n.s. \\
\hline Peak stress: & $1.3 \pm 0.9$ & $1.0 \pm 0.4$ & $1.2 \pm 0.4$ & $1.3 \pm 0.4$ & n.s. \\
\hline
\end{tabular}

In the tested patients heart rate increased by $73 \%$ and cardiac output by $85 \%$ during DSE. An increase in heart rate from $50-80 \%$ and cardiac output from $74-120 \%$ have been reported in five studies using the early low dose infusion $(20 \mu \mathrm{g} / \mathrm{kg} / \mathrm{min})[3,4,6,7]$. In three other studies [10-12] using the high dose $(40 \mu \mathrm{g} / \mathrm{kg} / \mathrm{min})$, heart rate increased from $69-90 \%$ and cardiac output increased from $88-110 \%$. As the increase in transvalvular gradients is dependent of flow, the differences in test conditions make comparison of data difficult.

In this study the hemodynamic characteristics of the St. Jude Regent bileaflet prostheses were examined in 66 patients with valve sizes from $19-25 \mathrm{~mm}$. As expected mean transvalvular gradients at rest and during stress decreased with increasing valve size. In the $21 \mathrm{~mm}$ sized prostheses mean pressure gradient increased from $9.2 \pm$ $6.6 \mathrm{mmHg}$ to $21.1 \pm 10.0 \mathrm{mmHg}$. These values are associated with a relatively mild transprosthetic gradient at rest and during peak stress. A few studies have previously compared various valve types based on stress tests. Slightly lower pressure gradients and slightly smaller EOA during stress, have been reported by Izzat et al in two studies in 1995 and 1996 [3,4]. These studies included 9 and 10 patients and were performed using bileaflet mechanical valves (Carbo Medics 19-21 mm valves and St. Jude $21 \mathrm{~mm}$ ). Contrary, a few studies using DSE in $21 \mathrm{~mm}$ sized prostheses have documented higher pressure gradients. Kadir et al [8] using the Sorin $21 \mathrm{~mm}$ valve, documented that mean transvalvular gradient increased from $15.6 \pm 5.3$ to $35.4 \pm 11.9 \mathrm{mmHg}$ during DSE. In another study [6] using Medtronic Intact aortic bioprotheses, mean transvalvular gradient increased from $19.1 \pm 5.1$ to $33.2 \pm 7.7 \mathrm{mmHg}$.

In our study the aortic valve area (EOA) remained relatively unchanged despite a significant increase in transprothetic gradients. This finding is in line with the majority of other studies $[3,4,6,9,12]$, and is possibly explained because mechanical components are less prone to accompany larger stroke volumes by increasing the EOA.
An EOAI greater than $0.85-0.9 \mathrm{~cm} 2 / \mathrm{m} 2$ has been suggested to minimize the effects of transprosthetic gradient and patient-to-prosthesis mismatch $[1,21]$. In our study the values for EOAI were above this limit in all sizes both at rest and during peak stress, indicating a favorable hemodynamic performance.

To our knowledge this study is one among the largest using DSE in the postoperative assessment patients following aortic valve replaement. In our experience DSE can be performed safely in all patients and maximum stress can be achieved in the majority of patients. Our study demonstrates excellent hemodynamic results for the St. Jude Medical Regent valve, even at small size, and a significant reduction in the left ventricular mass after one year.

\section{Conclusion}

The Dobutamine test induces a substantial stress, well suitable for echocardiographic assessment of prosthesis valve function and can be performed in the majority of the patients. The changes in pressure gradients add to the hemodynamic characteristics of the various valve sizes. In our patients the St. Jude Regent valve performed satisfactory at rest and under pharmacological stress situation.

\section{Acknowledgements}

We would like to thank Kristof Daems, Brussels, for performing the statistical analyses. This multicenter scientific follow-up study received a financial grant by the St. Jude Company.

\section{Author details}

'Oslo University Hospital, Rikshospitalet, PO box 4950, Nydalen, NO 0424 Oslo, Norway. ${ }^{2}$ University Hospital, Getingevaegen 4, SE 22185 Lund, Sweden. ${ }^{3}$ Blekingesjukhuset, SE 37181 Karlskrona, Sweden. ${ }^{4}$ Akademiska sjukhuset, SE 75185 Uppsala, Sweden. ${ }^{5}$ University Hospital, SE 70185 Örebro, Sweden. ${ }^{6}$ Norrlands Universitetssjukhus, SE 90185, Umeå, Sweden. ${ }^{7}$ St. Olavs University Hospital, PO Box 3250, Sluppen, NO 7006 Trondheim, Norway.

Authors' contributions

KA and JL. Svennevig were responsible for the study design. The final protocol was agreed on in a censensus meeting. All authors were involved 
in patients inclusion, treatment, follow-up and collection of data. All authors have read and approved the final manuscript.

\section{Competing interests}

The authors declare that they have no competing interests.

Received: 19 September 2011 Accepted: 19 December 2011 Published: 19 December 2011

\section{References}

1. Rahimtoola SH: The problem of valve prosthesis - patient mismatch. Circulation 1978, 58:20-4.

2. Taggart DP: Prosthesis patient mismatch in aortic valve replacement: possible but pertinent? Eur Heart J 2006, 27:644-6.

3. Izzat MB, Birdi I, Wilde P, Bryan AJ, Angelini GD: Evaluation of the hemodynamic performance of small CarboMedics aortic prostheses using dobutamine stress Doppler echocardiography. Ann Thorac Surg 1995, 60:1048-52.

4. Izzat MB, Birdi I, Wilde P, Bryan AJ, Angelini GD: Comparison of hemodynamic performance of St. Jude Medical and CarboMedics 21 $\mathrm{mm}$ aortic prostheses by means of dobutamine stress echocardiography. J Thorac Cardiovasc Surg 1996, 111:408-15.

5. Kadir I, Izzat MB, Birdi I, Wilde P, Angelini GD: Hemodynamics of St. Jude Medical prostheses in the small aortic root: in vivo studies using dobutamine Doppler echocardiography. J Heart Valve Dis 1997, 6:123-9.

6. Kadir I, Izzat MB, Wilde P, Reeves B, Bryan AJ, Angelini GD: Dynamic evaluation of the $21 \mathrm{~mm}$ Medtronic Intact aortic bioprostheses by dobutamine echocardiography. Ann Thorac Surg 1997, 63:1118-32.

7. Kadir I, Izzat MB, Birdi l, et al: Hemodynamic performance of the $21 \mathrm{~mm}$ St. Jude Biolmplant prostheses using dobutamine Doppler echocardiography. Am J Cardiol 1998, 81:599-603.

8. Kadir I, Wan IYP, Walch C, Wilde P, Bryan AJ: Angelini GD: Hemodynamic performance of the $21 \mathrm{~mm}$ Sorin Biocarbon mechanical aortic prosthesis using dobutamine Doppler echocardiography. Ann Thorac Surg 2001, 72:49-53.

9. Kadir I, Walch C, Wilde P, Bryan AJ, Angelini GD: Comparison of exercise and dobutamine echocardiography in the hemodynamic assessment of small size mechanical aortic valve prostheses. Eur J Cardiothorac Surg 2002, 21:692-7.

10. Zabalgoitia M, Kopec K, Abochamh DA, Oneschuk L, Herrera J, O Rourke RA: Usefulnes of dobutamine echocardiography in the hemodynamic assessment of mechanical prostheses in the aortic position. Am J Cardiol 1997, 80:523-6.

11. Hobson NA, Wilkinson GA, Wheeldon NM, Lynch J: Hemodynamic performance of the Ultracor and Carpentier-Edwards prostheses using exercise and dobutamine stress echocardiography. J Heart Valve Dis 2005, 14:838-44.

12. Minardi G, Manzara C, Creazzo V, Casali D, Pulignaro G, Musumeci F: Evaluation of 17 med mer St. Jude Medical Regent prosthetic valves by rest and dobutamine stress echocardiography. J Cardiothorac Surg 2006, 1:1-27.

13. Bach DS, Goldbach M, Sakwa MP, Petracek M, Errett L, Mohr F: SJM Regent study group. Hemodynamic and early performance of the St. Jude Medical Regent aortic valve prostheses. J Heart Valve Dis 2001, 10:436-42.

14. Pellikka PA, Roger VL, Oh JK, Miller FA, Seward JB, Tajik AJ: Stress echocardiography. Part II. Dobutamine stress echocardiography: technics, implementation, clinical applications and correlations. Mayo Clin Proc 1995, 70:16-27.

15. Picano E: Stress echocardiography. BERLIN, Heidelberg, New York: Springer; 1997, 95-9.

16. Sicari R, Nihoyannopolos P, Evangelista A, et al: Stress echocardiography expert consensus statement: European Association of Echocardiography (EAE) (a registered branch of the ESC). Eur J Echocardiography 2008, 9:415-37.

17. Kameran M, Teague SM, Finkelhor RS, Dawson N, Bahler RC: Prognostic value of dobutamine stress echocardiography in patients referred because of suspected coronary artery disease. Am J Cardiol 1995, 76:887-91.

18. Schwammental E, Vered Z, Rabinowitz B, Kaplinsky E, Feinberg S: Stress echocardiography beyond coronary artery disease. Eur Heart J 1997, 18(suppl D):30-37.
19. Lin SS, Roger VI, Pascoe R, Seward JB, Pellika PA: Dobutamine stress Doppler hemodynamics in patients with aortic stenosis: feasability, safety and surgical correlations. Am Heart J 1998, 136:940-2.

20. Wu WC, Ireland LA, Sadaniantz A: Evaluation of aortic valve disorders using stress echocardiography. Echocardiography 2004, 21:459-66.

21. Rezaian GR, Aghasadeghi K, Kojuri J: Evaluation of the hemodynamic performance of St. Jude mitral prostheses: a pilot study by dobutamine stress Doppler echocardiography. Angiology 2005, 56:81-6.

doi:10.1186/1749-8090-6-163

Cite this article as: Offstad et al:: The Scandinavian multicenter hemodynamic evaluation of the SJM Regent aortic valve. Journal of Cardiothoracic Surgery 2011 6:163.

\section{Submit your next manuscript to BioMed Central and take full advantage of:}

- Convenient online submission

- Thorough peer review

- No space constraints or color figure charges

- Immediate publication on acceptance

- Inclusion in PubMed, CAS, Scopus and Google Scholar

- Research which is freely available for redistribution

Submit your manuscript at www.biomedcentral.com/submit
Biomed Central 